\title{
Comparação entre Métodos Iterativos na Resolução de Sistemas Não-Lineares
}

\author{
Elienai Alves de Souza Gustavo Benitez Alvarez Diomar Cesar Lobão \\ Modelagem Computacional em Ciência e Tecnologia - PPG-MCCT, \\ 27225-125, Vila Santa Cecília, Volta Redonda, RJ \\ E-mail: profelienai.vr@gmail.com, benitez.gustavo@gmail.com, lobaodiomarcesar@yahoo.ca
}

\section{RESUMO}

Os sistemas de equações não-lineares surgem em muitos problemas da engenharia, matemática, robótica e ciências da computação devido a maioria dos sistemas físicos serem não-lineares. Considere o sistema de equações não-lineares:

$$
\left\{\begin{array}{c}
f_{1}\left(x_{1}, x_{2}, \ldots, x_{n}\right)=0 \\
f_{2}\left(x_{1}, x_{2}, \ldots, x_{n}\right)=0 \\
\vdots \\
f_{n}\left(x_{1}, x_{2}, \ldots, x_{n}\right)=0
\end{array} \Longrightarrow F(X)=0,\right.
$$

sendo $f_{1}, \cdots, f_{n} n$ funções não-lineares de $n$ variáveis e $F$ uma função vetorial com $X \in \mathbb{R}^{n}$.

Solucionar este sistema consiste em determinar os $X$ que satisfaçam (1), sendo possível em raros casos obter soluções exatas. Em geral, podemos obter soluções aproximadas utilizando métodos iterativos do tipo $X^{(k+1)}=G\left(X^{(k)}\right)$, onde $X^{(k)}$ é a solução aproximada na k-ésima iteração. Neste trabalho estudaremos as bacias de atração e as taxas de convergência dos métodos de Newton [2]; LevenbergMaquardt [8]; Broyden [1]; Gradiente [3]; Steffensen [6]; Halley [7] e Householder [5].

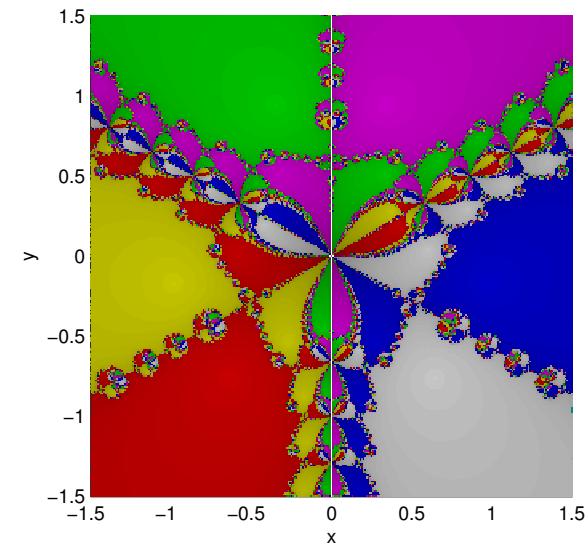

(a) Newton

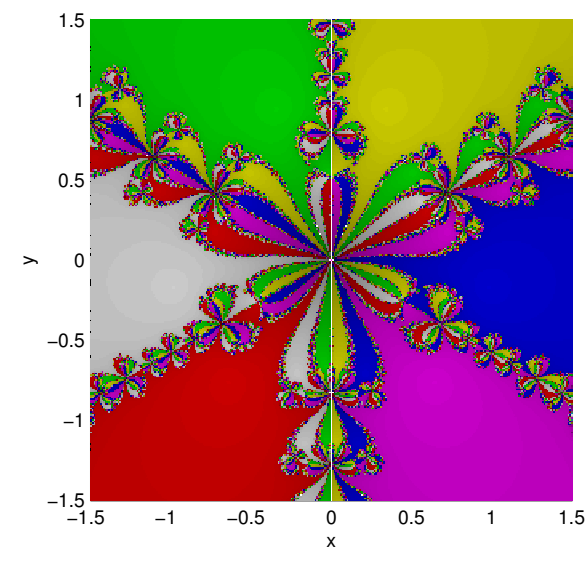

(b) Householder

Figura 1: Bacias de atração para o problema $p(z)=z^{6}+i z^{3}-1=0$ com $z=x_{1}+i x_{2}$, no domínio quadrado $-1.5 \leq x_{1} \leq 1.5 \mathrm{e}-1.5 \leq x_{2} \leq 1.5$, com resolução de $301 \times 301$ pixels, numero máximo de iterações 80 e critério de parada $\left|f_{1}\left(X^{(k+1)}\right)\right|<\varepsilon$ e $\left|f_{2}\left(X^{(k+1)}\right)\right|<\varepsilon \operatorname{com} \varepsilon=10^{-12}$.

É conhecido que as bacias de atração de alguns destes métodos apresentam uma estrutura fractal como mostrado na figura 1. Existem resultados teóricos que garantem uma taxa de convergência específica para cada método. Entretanto, estas taxas podem apresentar variações dependendo de fatores 


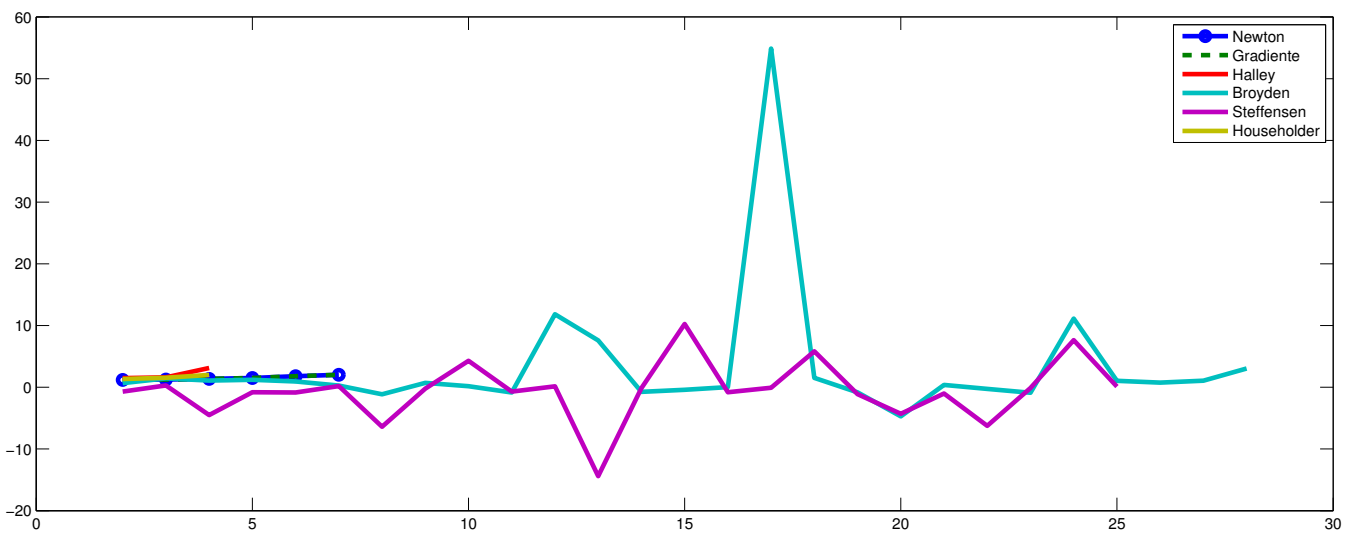

Figura 2: Taxas de convergência para o problema $p(z)=z^{6}+i z^{3}-1=0$ com $z=x_{1}+i x_{2}$, onde a ordenada é $\log \left(\left\|X^{(k+1)}-X^{*}\right\|-\left\|X^{(k)}-X^{*}\right\|\right) / \log \left(\left\|X^{(k)}-X^{*}\right\|-\left\|X^{(k-1)}-X^{*}\right\|\right)$, a abscissa é k, $X^{*}$ é a solução aproximada obtida na última iteração por cada método, $X^{(0)}=(-1.5,-1.5)$ e $\|\cdot\|$ é a norma euclidiana.

como a escolha inicial $\left(X^{(0)}\right)$ perto da raiz, regularidade das funções $f_{1}, \cdots, f_{n}$, entre outros, como mostrado na figura 2.

Os resultados apresentados acima mostram a necessidade de estabelecer critérios que permitam avaliar melhor o desempenho destes métodos. Embora existam alguns trabalhos indicando vantagens e desvantagens destes métodos, atualmente, escolher o método mais apropriado para resolver um determinado sistema de equações não-lineares é uma tarefa difícil. Em trabalhos futuros, pretendemos aplicar este estudo para problemas de engenharia que recaem em sistemas de equações não-lineares.

Palavras-chave: Métodos Iterativos, Sistemas de Equações Não-Lineares, Convergência, Fractais

\section{Referências}

[1] C.G. Broyden, On the Discovery of the "good Broyden" Method, Math. Program., 87 (2000), n 2, 209-213.

[2] S.D. Conte, C. De Boor, "Elementary Numerical Analysis, an Algorithmic Approach", McGrawHill Book Company, São Paulo, 1980.

[3] B.P. Demidovich, "Computational Mathematics", Translated from the Russian by George Yankovsky, MIR Publishers, Moscow, 1981.

[4] D.M. Gay, Some Convergence Properties of Broyden's Method, SIAM J. Numer. Anal., 16 (1979), $\mathrm{n}^{\mathrm{o}} 4,623-630$.

[5] M.A. Hafiz, S.M. Bahgat, Modified of Householder Iterative Method for Solving Nonlinear Systems, J. Math. Comput. Sci., 2 (2012), nº 5, 1200-1208.

[6] P. Henrici, Elements of Numerical Analysis, John Wiley \& Sons Ltd, New York, 1964.

[7] T. Park, J. Ji, K. Hee Ko, A Second Order Geometric Method for Ray/Parametric Surface Intersection, Comput. Aid. Geom. Des., 30 (2013), nº 8, 795-804.

[8] B.T. Polyak, Newton's method and its use in optimization, Eur. J. Oper. Res., 181 (2007), n 3, 1086-1096. 